\title{
Quadratur des Kreises?
}

\section{Eine ökologische Innovationspolitik kann prinzipiell geeignet sein, Ökonomie und Ökologie zu versöhnen und stellt zugleich eine mögliche Antwort auf das häufig diagnostizierte Problem der internationalen Wettbewerbsfähigkeit dar. Allerdings erfordert dies eine Überwindung der bisherigen Technikzentrierung und einen radikalen Umbau des deutschen Innovationssystems.}

In:

Von Kurt Hübner

m Zeichen globalisierter Märkte für Waren,

Dienstleistungen, Kapital und Arbeit hat sich der internationale Konkurrenzdruck für alle ökonomischen Akteure enorm erhöht. Internationale Wettbewerbsfähigkeit ist auf der wirtschafts- und sozialpolitischen Tagesordnung ganz nach oben geriickt. Scheint diese Priorität in den OECDGesellschaften heute relativ einhellig akzeptiert zu sein, so sind die Wege zu einer Verbesserung internationaler Wettbewerbsfähigkeit doch heftig umstritten. Grob vereinfachend gesprochen, aber dennoch keineswegs an der Sache vorbeigehend, lassen sich zwei Pole in der Debatte unterscheiden. Die eine Position diagnostiziert den Kern des Wettbewerbsfähigkeitsproblems auf der Kostenseite. $\mathrm{Zu}$ hohe Produktionskosten, insbesondere seitens des Faktors Arbeit, belasten danach die preisliche Konkurrenzähigkeit nationaler Hersteller. Die wenigstens relative Senkung der direkten wie der indirekten Produktionskosten werden als adäquate Medizin empfohlen. Die andere Position diagnostiziert das Wettbewerbsfähigkeitsproblem auf der Produkt- und Prozeßseite. Das Verharren auf traditionellen Märkten ,alter" Produkte und mangelnde Anpassung an neue Produktionsformen werden als Kern des Problems angesehen, und entsprechend Innovationen als therapeutisches Allheilmittel empfohlen.

Beide Politikvarianten können zu einer Senkung der Lohnstiickkosten und dadurch zu einer Verbesserung der internationalen Wettbewerbsfähigkeit führen. Während die Kostenvariante den unmittelbaren Nachteil hat, daß die Gruppe der Lohnabhängigen die Bürde der Anpassung zu tragen hat, wird der zweiten Variante der Vorteil zugerechnet, daß neue Produkte sowie Steigerungen der Arbeitsproduktivität erhöhte Verteilungsspielräume und mehr Beschäftigung zeitigen können. Umgekehrt verhalten sich die Vorteile und Nachteile unter dem Gesichtspunkt des Zeithorizontes. Während nämlich die Innovationsstrategie eher mittel- und langfristig
Erfolge zeitigen kann, wirkt die Kostenstrategie verhältnismäßig schnell. Unter Bedingungen globalisierter Geld- und Finanzmärkte, die schnelle Erträge der Unternehmen bevorzugt belohnen, ist dies ein nicht zu unterschätzender Vorteil. Gerade diese Eigenschaft dürfte erklären, warum trotz aller Innovationsrhetorik die Kostenstrategie eine hervorgehobene Rolle spielt. Die Sicherstellung längerfristigen Wachstums ist freilich auf Neuerungen angewiesen. Daß der innovatorische Prozeß eine Schlüsselgröße für das Wachstum von Volkswirtschaften darstellt, ist zwar spätestens seit den Zeiten von Joseph Schumpeter in der ökonomischen Zunft bekannt. Es bedurfte allerdings erst der strikten ökonomischen Modellierung, daß sich diese Erkenntnis unter dem Rubrum der neven Wachstumstheorie hat nachhaltiges akademisches Gehör verschaffen können. Die Konzeptualisierung von technologischem Fortschritt als endogener Größe des Wachstumsprozesses hat viele innovationspolitische Facetten hervorgebracht, von denen die Variante einer ökologischen Innovationspolitik die größte Faszination ausstrahlt. Eine solche Ausformung von Innovationspolitik scheint die gerne versprochene Versöhnung von Ökologie und Ökonomie wahrmachen zu können. Nachhaltige Technikentwicklung, Eröffnung neuer Märkte und damit Absatzmöglichkeiten und Schaffung neuer Einkommen sowie nachhaltiges Wachstum würden durch ökologisch-intelligente ökonomische Neuerungen auf elegante Weise zusammengefuihrt.

\section{Nevorientierung notwendig}

Abseits von den Gesetzen der Thermodynamik spricht analytisch viel für eine solche Spielart der Innovationspolitik. Obwohl aus einschlägigen Untersuchungen bekannt ist, daß der Einbau ökologischer Kriterien in die Investitionsentscheidungen privater Unternehmen zu relativen Einbußen in den Zuwachsraten der Gesamtfaktorproduktivität führt, ist dies dennoch kein schlagendes Argument gegen eine solche strategische Neuori- entierung. Anders nämlich als der einschlägige beratungsprofessionelle Sachverstand behauptet, wonach die Lösung ökologischer Probleme „,her die optimale Anwendung vorhandenen Wissens unter umweltspezifischen Fragestellungen als die Entwicklung von Spitzentechnologien" verlange (1), erfordert eine ökologische Innovationspolitik einen radikalen Paradigmenwechsel in der Forschungs- und Technologiepolitik. Dies im privaten wie im staatlichen Bereich.

Ökologische Innovationspolitik, will sie ökonomische wie ökologische Erfolge zeitigen, muß die bisherige Technikzentrierung von F\&E überwinden und sich einem gesellschaftlichen Effizienzkriterium aussetzen, das die positiven wie negativen externen Effekte privater ökonomischer Handlungen einbezieht. Dies erfordert einen grundsätzlichen Wandel in der ökonomischen Rechnungsfiihrung ebenso wie eine konsequente Ausweitung des Innovationsbegriffs auf die Konsumtionsseite gesellschaftlicher Reproduktion. Längst nicht jede Innovation, die sich produktionsseitig für die Hersteller von Produkten lohnt, ist ökologisch wie auch makroökonomisch sinnvoll. Im Zeitalter einer Internalisierung negativer externer Effekte ist ein solcher Wandel auch unter Gesichtspunkten der Wettbewerbsfähigkeit in höchstem Maße rational. Ein solcher Wandel in Richtung einer ökologisch verträglichen und wettbewerbssteigernden Innovationspolitik erfordert aber nicht nur einen Paradigmenwechsel. Erforderlich ist vor allem ein radikaler Umbau des bundesdeutschen Innovationssystems unter Einbezug aller Akteure. Dies mag politisch ein Wunschtraum sein, und vielleicht auch bleiben. Aus einer ökonomischen und ökologischen Perspektive wäre es freilich an der Zeit, diesen Traum in die Tat umzusetzen.

\section{Anmerkung}

(1) Gemeinschaftsgutachten "Zur technologischen Leistungsfähigkeit Deutschlands". Zusammenfassender Endbericht 1998, S. 57. Im Internet abrufbar unter www.zew.de

\section{Der Autor}

Dr. Kurt Hübner ist Professor an der Fachhochschule für Wirtschaft Berlin und zur Zeit Gasiprofessor an der Vork University in Toronto.

Kontakt: York University, The Canadian Centre for German And European Studies, 230 York Lanes 4700 Keele Street, Toronto, Ontario M3J IP3, Kanada. Tel. 0041-6-736-2100, Fax - 5696 , E-Moil: khuebner@yorku.ca 
(c) 20I0 Authors; licensee IÖW and oekom verlag. This is an article distributed under the terms of the Creative Commons Attribution Non-Commercial No Derivates License (http://creativecommons.org/licenses/by-nc-nd/3.o/), which permits unrestricted use, distribution, and reproduction in any medium, provided the original work is properly cited. 